\title{
Time course of cochlear electrophysiology and morphology after combined administration of kanamycin and furosemide
}

\author{
Huib Versnel *, Martijn J.H. Agterberg, John C.M.J. de Groot, \\ Guido F. Smoorenburg, Sjaak F.L. Klis \\ Department of Otorhinolaryngology, Rudolf Magnus Institute of Neuroscience, University Medical Center Utrecht, \\ P.O. Box 85500, 3508 GA Utrecht, The Netherlands
}

Received 3 December 2006; received in revised form 5 March 2007; accepted 6 March 2007

Available online 31 March 2007

\begin{abstract}
In animal models of deafness, administration of an aminoglycoside in combination with a loop diuretic is often applied to produce a rapid loss of cochlear hair cells. However, the extent to which surviving hair cells remain functional after such a deafening procedure varies. In a longitudinal electrocochleographical study, we investigated the variability of cochlear function between and within guinea pigs after combined administration of kanamycin and furosemide. Concurrently, histological data were obtained at 1, 2, 4 and 8 weeks after deafening treatment. The main measures in our study were compound action potential (CAP) thresholds, percentage of surviving hair cells and packing density of spiral ganglion cells (SGCs). One day after deafening treatment, we found threshold shifts widely varying among animals from 0 to $100 \mathrm{~dB}$. The variability decreased after 2 days, and in 18 out of 20 animals threshold shifts greater than $55 \mathrm{~dB}$ were found 4-7 days after deafening. Remarkably, in the majority of animals, thresholds decreased by up to $25 \mathrm{~dB}$ after $7 \mathrm{days}$ indicating functional recovery. As expected, final thresholds were negatively correlated to the percentage of surviving hair cells. Notably, the percentage of surviving hair cells might be predicted on the basis of thresholds observed one day after deafening. SGC packing density, which rapidly decreased with the period after deafening treatment and correlated to the percentage of surviving inner hair cells, was not a determining factor for the CAP thresholds.
\end{abstract}

(C) 2007 Elsevier B.V. All rights reserved.

Keywords: Deafness; Ototoxicity; Recovery; Cochlear hair cells; Spiral ganglion cells; Electrocochleography

\section{Introduction}

Various animal models are used to study cochlear dysfunction or sensorineural deafness. For instance, congenitally deaf animals can be bred or the cochlea can be mechanically destroyed. More often, however, ototoxic

Abbreviations: CAP, compound action potential; CM, cochlear microphonics; IHC, inner hair cell; OHC, outer hair cell; SGC, spiral ganglion cell.

* Corresponding author. Tel.: +31 30 2507564; fax: +31 302522627.

E-mail addresses: h.versnel@umcutrecht.nl (H. Versnel), M.J.H. Agterberg@umcutrecht.nl (M.J.H. Agterberg), J.C.M.J.deGroot@ umcutrecht.nl (J.C.M.J. de Groot), guido.smoorenburg@wanadoo.fr (G.F. Smoorenburg). drugs are used for this purpose. A common ototoxic procedure, first described by West et al. (1973), is a single administration of an aminoglycoside (e.g., kanamycin) in combination with a loop diuretic (ethacrynic acid or furosemide). This method has been applied in studies investigating neural degeneration (Webster and Webster, 1981; $\mathrm{Xu}$ et al., 1993), neurotrophic treatment to preserve the cochlear nerve (Staecker et al., 1996; Miller et al., 1997; Gillespie et al., 2003), electrical stimulation with a cochlear implant (Lousteau, 1987; Mitchell et al., 1997), and hair cell regeneration (Izumikawa et al., 2005). However, the extent to which hair cells remain functional after the deafening procedure, i.e., the actual extent of deafness, can vary. A significant variability among animals was demonstrated by Nourski et al. (2004), who found that $12 \mathrm{~h}$ after 
administration of kanamycin in combination with ethacrynic acid 4 out of 6 guinea pigs showed the intended loss of cochlear response (to click stimuli of $80 \mathrm{~dB}$ above normal threshold) and 2 out of 6 animals showed a substantial response. For this reason, auditory evoked potentials are often used to assess the result of the deafening procedure. For instance, using auditory brainstem responses in an experiment involving preservation of the nerve after hair cell loss, Shepherd et al. (2005) reported exclusion of animals because of insufficient increases of thresholds. A deafness check is especially relevant when long-term and/or expensive studies involving neurotrophic treatment are performed. It is then assumed that the condition of the cochlea does not change significantly after the animal passes the electrophysiological deafness check. However, variability within an animal might exist. For instance, Aran and Darrouzet (1975) demonstrated recovery from a 40-dB loss in the second week after 8-day treatment with kanamycin. Also, Klis et al. $(2000,2002)$ found substantial recovery over periods up to 3 weeks after long-term cisplatin treatment.

In the present study, we examined differences between animals and the within-animal changes during a period of up to 8 weeks after administration of kanamycin in combination with furosemide. In guinea pigs with a permanent round window electrode, cochlear potentials in response to click and tone stimuli were recorded. These recordings were performed frequently during the first week after deafening and on a once-weekly basis thereafter. Clicks were included in the stimulus set as these are commonly used for electrophysiological checks (e.g., auditory brainstem responses). We measured the time course of the compound action potential (CAP) thresholds in order to address changes over time and differences between animals. The CAP thresholds were related to histological measures, i.e., the percentage of surviving hair cells and the spiral ganglion cell (SGC) packing density. A specific issue was the predictability of hair cell loss on the basis of thresholds during the first week after deafening.

\section{Materials and methods}

\subsection{Animals and surgery}

Twenty-five female albino guinea pigs (strain: Dunkin Hartley; supplier: Harlan Laboratories, Horst, The Netherlands) weighing 270-600 g were used. Animals had free access to food and water, and were kept under standard laboratory conditions. All experimental procedures were approved by the University's Committee on Animal Research (DEC-UMC \#03.04.036).

The animals were injected with a non-ototoxic antibiotic (chloramphenicol sodium succinate; $60 \mathrm{mg} / \mathrm{kg}$, im) and anesthetized with $40 \mathrm{mg} / \mathrm{kg}$ ketamine hydrochloride $\left(\right.$ Ketanest $^{\circledR}$, im) and $10 \mathrm{mg} / \mathrm{kg}$ xylazine hydrochloride (Sedamun ${ }^{\circledR}$, im). Local anesthetic $(1 \%$ lidocaine) was used in areas to be incised. An electrode consisting of a stainless steel wire with a gold-ball tip was positioned in the round window niche of the right cochlea and sealed into the bulla (for details, see Klis et al., 2000, 2002). Stainless steel screws were inserted through the skull near the bregma to be used as reference and ground electrodes.

\subsection{Deafening procedure}

At least 2 weeks after electrode implantation, allowing for recovery from surgical trauma, 20 animals were concomitantly treated with kanamycin and furosemide (West et al., 1973). After electrocochleography, the animals were anaesthetized with ketamine $(40 \mathrm{mg} / \mathrm{kg}$, im) and xylazine $(10 \mathrm{mg} / \mathrm{kg}$, im). Kanamycin sulphate in isotonic saline $(400 \mathrm{mg} / \mathrm{kg}$, sc) was administered and, subsequently, the external jugular vein was exposed and cannulated and, 15-60 min after kanamycin injection, furosemide $\left(100 \mathrm{mg} / \mathrm{kg}\right.$, Centrafarm $\left.{ }^{\circledR}\right)$ was slowly infused. The dose of kanamycin is the same as in numerous studies that apply kanamycin in combination with ethacrynic acid (e.g., Staecker et al., 1996; Miller et al., 1997; Nourski et al., 2004) or furosemide (e.g., Gillespie et al., 2003; Shepherd et al., 2005; Wise et al., 2005) for deafening purposes. The dose of furosemide is the same as applied in the latter studies.

In five control animals a sham procedure was performed with isotonic saline without the ototoxic drugs.

\subsection{Electrocochleography and acoustic stimuli}

Recordings were performed as described in detail by Hamers et al. (2003). The animal, positioned in a soundproof chamber, was awake; it was lightly restrained in a custom-made tight cloth jacket with the eyes covered and the right pinna and ear canal exposed. Sound generation and ECoG recordings were controlled by a personal computer and a DT3010/32 data acquisition board (Data translation). Acoustic stimuli were tone bursts of 2, 4, 8, 11.3 and $16 \mathrm{kHz}$ with a duration of $8 \mathrm{~ms}(1 \mathrm{~ms}$ rise/fall

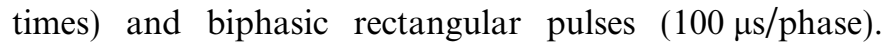
The sound stimuli were presented in an open-field configuration with a Fane tweeter (J-104) positioned at $10 \mathrm{~cm}$ from the pinna. Using a pair of attenuators (PA4, Tucker-Davis Technologies), sound levels were varied from about $100 \mathrm{~dB}$ SPL (frequency dependent) down to below threshold in 10-dB steps. The sound levels were calibrated by a sound level meter (Brüel \& Kjaer, 2203) and a 1-in. condenser microphone (Brüel \& Kjaer, 4132). Stimuli were presented with alternating polarity at $99 \mathrm{~ms}$ intervals. The responses were amplified (5000×; EG\&G Instruments model 5113$)$, bandpass-filtered $(1 \mathrm{~Hz}-30 \mathrm{kHz})$, and sampled at $33 \mathrm{kHz}$.

The CAP and summating potential (SP) were obtained by adding the responses evoked by stimuli of opposite polarity, cochlear microphonics $(\mathrm{CM})$ by subtracting these 
responses. The amplitude of the CAP was defined as the difference between the SP and the $\mathrm{N}_{1}$ peak. The amplitude of the CM was measured peak-to-peak. Response thresholds were assessed by deriving an iso-response level from the input/output curves (Fig. 1), specifically, the sound level at which the amplitude is $3 \mu \mathrm{V}$. In general, the CAP was used to assess the threshold.

\subsection{Histology}

Immediately after the final ECoG measurements, the left and right cochleas were fixed by intralabyrinthine perfusion with a tri-aldehyde fixative. Cochleas were processed as described in detail by De Groot et al. (1987). Cochleas were embedded in toto in Spurr's low-viscosity resin and divided into two halves along a standardized midmodiolar plane (Van Ruijven et al., 2004). Semithin $(1 \mu \mathrm{m})$ sections were stained with methylene blue and azur II in sodium tetraborate and examined with a Zeiss Axiophot light microscope. The number of inner hair cells (IHCs) and outer hair cells $(\mathrm{OHCs})$ was counted in each individual transection of the respective half-turn $(\mathrm{b} 1, \mathrm{~b} 2, \mathrm{~m} 1, \mathrm{~m} 2, \mathrm{a} 1, \mathrm{a} 2, \mathrm{a} 3$; see Fig. 6A), as described in Van Ruijven et al. (2004). Digitized light-microscopical images of the spiral ganglia from each individual transection were analysed using the software program NIH Image, version 1.63 (http:// www.rsb.info.nih.gov/nih-image). The bony boundaries of Rosenthal's canal were outlined using a pressure-sensitive stylus on a digitizer tablet, and its cross-sectional area was calculated. The number of SGC perikarya was counted in each transection of Rosenthal's canal. SGC packing density was calculated by dividing the number of perikarya by the cross-sectional area (in $\mathrm{mm}^{2}$ ).

\subsection{Experimental groups}

We used four experimental groups and one control group, with each group consisting of five guinea pigs. Experiments were performed in several series of three to six animals each; the assignment of an animal to an experimental group was random with the restriction that in each series of experiments the experimental groups were evenly represented. The four experimental groups were sacrificed for histological preparation after four different periods following the deafening procedure: 1, 2, 4 and 8 weeks. The control group was sacrificed 8 weeks after the sham treatment.

In all animals, ECoG was performed immediately before deafening and at the following times after deafening: immediately after (day 0), after 1 day, and after 2, 4 and 7 days. Subsequently, recordings were continued once-weekly (up to 2,4 or 8 weeks) in so far the animals were not sacrificed for histology.

\section{Results}

\subsection{Time course in the first week after deafening}

Twenty guinea pigs were treated with kanamycin and furosemide in order to cause severe or profound hearing loss. One day after the treatment, the differences of the effect of the deafening treatment among these 20 animals was marked, which is illustrated in Figs. 1 and 2.

Fig. 1 shows input/output characteristics of CAPs and $\mathrm{CMs}$ for two different guinea pigs at one time before and at various times after the deafening treatment. In both animals, immediately after deafening, the CAP curves showed

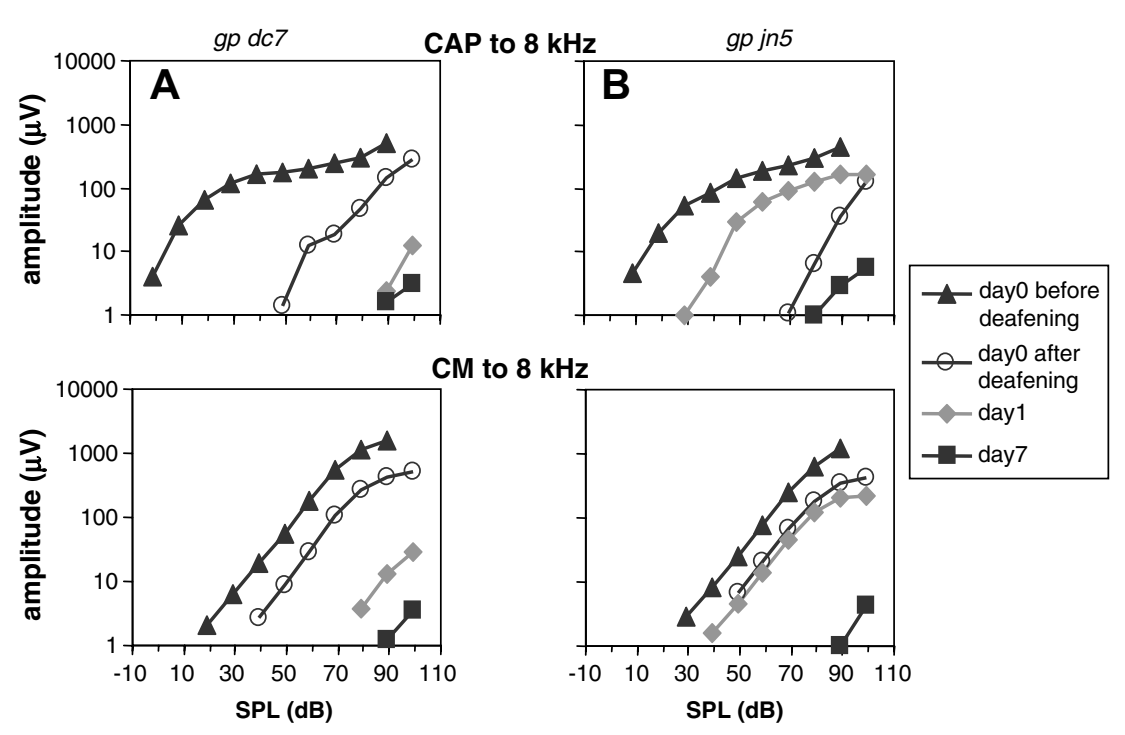

Fig. 1. Input/output curves of CAP (top) and CM (bottom) to an 8-kHz tone pre- and three times post-deafening: immediately before, immediately after, 1 day after and 7 days after deafening. (A) Input/output curves in guinea pig $d c 7$. The curves monotonically shift with time after deafening towards higher stimulus levels. (B) Input/output curves in guinea pig jn5. One day after deafening, the CAP curves show partial recovery, whereas the CM curves show a small decline. 

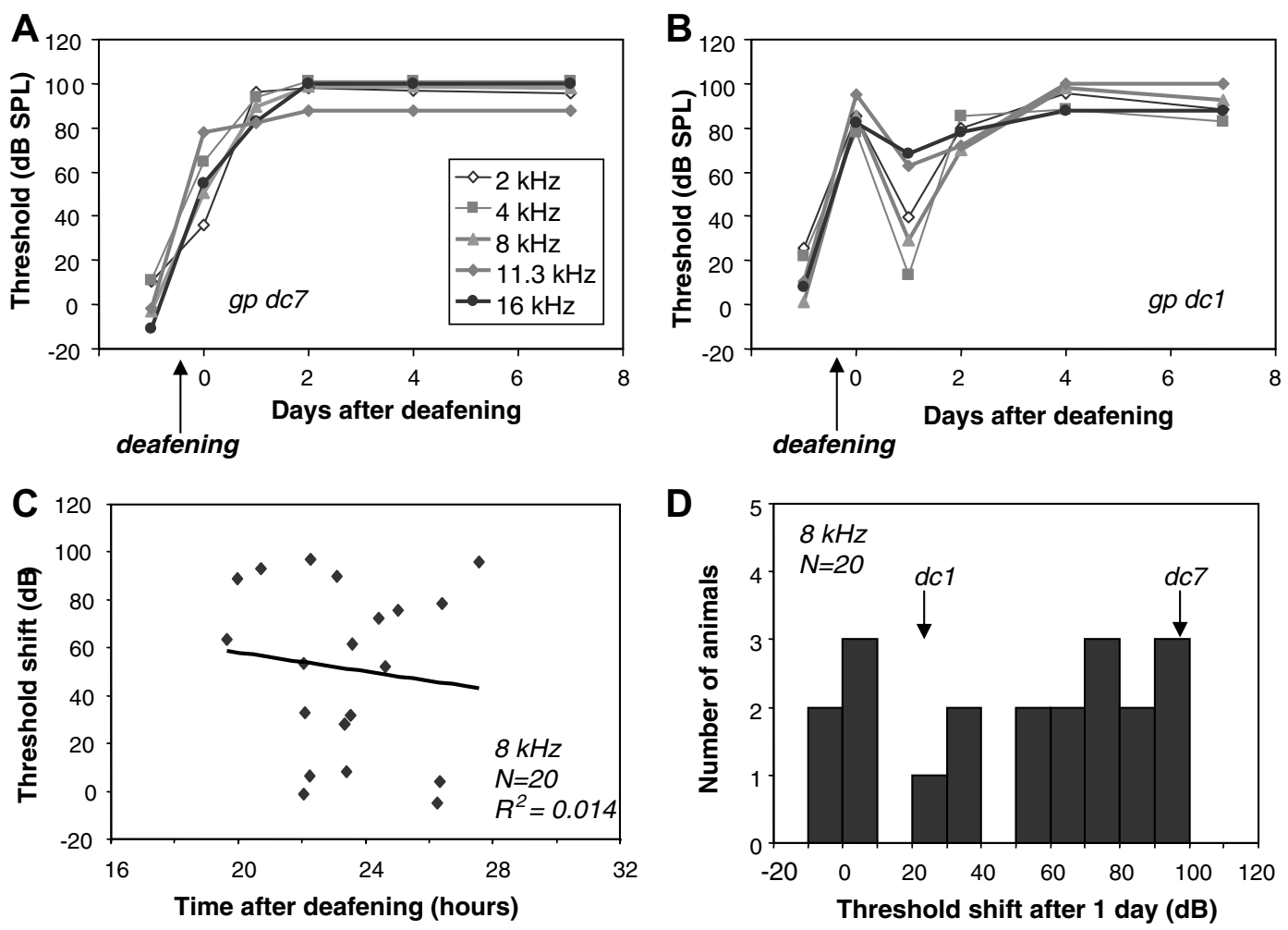

Fig. 2. (A, B) Time courses of CAP thresholds up to 7 days for five different tone frequencies $(2-16 \mathrm{kHz})$ in two guinea pigs. The CAP thresholds before deafening were recorded on day 0 , just before the deafening surgery. In (A) the thresholds at $4,11.3$ and $16 \mathrm{kHz}$ reached ceiling values from day 2 to day 7 . (C) Threshold shift at "one day" after deafening versus the actual 1-day period in hours. (D) Distribution of threshold shifts for $8 \mathrm{kHz}$ at 1 day after deafening.

a large shift to higher levels (about $60 \mathrm{~dB}$ ), whereas the $\mathrm{CM}$ curves showed only a small shift (about $15 \mathrm{~dB}$ ); these immediate effects were found in all deafened animals. Large differences between these two examples were seen on day 1 : in $d c 7$ (Fig. 1A) the CAP curve continued to worsen (by an additional $40 \mathrm{~dB}$ ), but in $j n 5$ (Fig. 1B) the CAP curve ameliorated (by $40 \mathrm{~dB}$ ). Although this recovery was not seen in the $\mathrm{CM}$ curves, the development of the CM curves differed substantially between the two animals in that the shift in $d c 7(40 \mathrm{~dB})$ was significantly greater than in $j n 5(3 \mathrm{~dB})$. This difference between $\mathrm{CM}$ responses concurred with the difference between CAP responses. Across all animals, the $\mathrm{CM}$ threshold shifts at day 1 correlated strongly to the CAP threshold shifts at day 1 (linear regression, $R^{2}>0.75, p<0.0001$; for each tone frequency used). Seven days after deafening the responses had almost disappeared in both animals; only at the highest stimulus levels (about $100 \mathrm{~dB}$ SPL) small CAP and CM waveforms $(<10 \mu \mathrm{V})$ were found. The great difference between the animals at day 1 was not apparent at day 7 .

The time courses of thresholds that are shown in Fig. 2 for two individual guinea pigs, reflect the great difference in thresholds between animals at day 1 after deafening. In animal $d c 7$ (Fig. 2A) the thresholds were high after 1 day for all frequencies and continued to shift the second day. In animal $d c 1$ (Fig. 2B) the thresholds were low at day 1, in particular for the lower frequencies $(2-8 \mathrm{kHz})$, but sub- stantially increased the second day (by $40-70 \mathrm{~dB}$ ) and continued to increase until day 4 . The frequency dependence of the thresholds at day 1 was observed in most animals.

Considering the large changes within two days as illustrated in Fig. 2B, it is conceivable that the thresholds at day 1 critically depend on the precise period between deafening and recording. Across all animals, the recordings at day 1 were performed between 20 and $28 \mathrm{~h}$ after deafening. We found that over this time interval, the threshold shift was not correlated with the actual period (Fig. 2C). This indicates that probably recovery occurred before $20 \mathrm{~h}$ and subsequent decline after $28 \mathrm{~h}$. The threshold shifts at day 1 were widely, and rather uniformly, distributed from 0 to $100 \mathrm{~dB}$ (Fig. 2C and D). This distribution moved towards large threshold shifts with increasing stimulus frequency (about $10 \mathrm{~dB}$ per octave) while the spread was similar across frequencies with distribution's standard deviations of $26-36 \mathrm{~dB}$.

The CAP and CM threshold shifts found after one day were not correlated to the immediate threshold shifts (linear regression across all animals, $R^{2}<0.2, p>0.1$ ) for either frequency used, apart from one condition (CM at $16 \mathrm{kHz}: R^{2}=0.3, p<0.05$ ). Finally, the weight of the animal, which partly reflects its age, was not a significant factor determining the threshold shifts after one day $\left(R^{2}<0.2\right.$, $p>0.05)$. 


\subsection{Thresholds 7 days after deafening}

The examples in Figs. 1 and 2 showed that thresholds at 7 days after deafening were sufficiently high according to the aim of a deafening treatment (shifts $>50-60 \mathrm{~dB}$, Mitchell et al., 1997; Shepherd et al., 2005). We found that in 18 out of 20 animals threshold shifts at day 7 were larger than $55 \mathrm{~dB}$ for clicks (Fig. 3B). Threshold shifts in the other two animals were small ( $<35 \mathrm{~dB}$ for clicks), which would be considered insufficient for a deafness model. These two animals have been excluded from various analyses in following sections of this report, since we are particularly interested in variability between and within animals with high thresholds.

Fig. 3 shows that the threshold shifts at day 7 were significantly correlated to the threshold shift at day 1 (linear regression, $p<0.01)$. This was found at all frequencies applied (in Fig. 3A: 2 kHz) and clicks (Fig. 3B).

\subsection{Long-term time courses of thresholds}

Unexpectedly, we found significant threshold decreases after 7 days indicating a recovery of cochlear function. Fig. 4 shows two examples of time courses where the threshold decreased during the second week after deafening. In one example (jn4, Fig. 4A), where the first-week time course was characterized by high thresholds at day 1 , the thresholds decreased by about $10 \mathrm{~dB}$ at the higher frequencies $(8-16 \mathrm{kHz})$ in the second week. In the second example (jn5, Fig. 4B), where the first-week time course was characterized by relatively low thresholds at day 1 , the thresholds decreased during the second week at all frequencies used $(2-16 \mathrm{kHz})$ by $5-25 \mathrm{~dB}$. In fact, this decrease already started after day 4 . After day 14 , a slow increase of thresholds was observed in jn4 (Fig. 4A), which was monitored for up to 56 days. This late decline starting after 2-4 weeks was found in 3 out of 5 animals that were monitored during 8 weeks. Linear regression analysis shows that averaged across the five animals the threshold increase from 4 to 8 weeks was small but significant $(2 \mathrm{~dB} /$ week, $R^{2}>0.75, p<0.05$; frequencies 2,4 , and $8 \mathrm{kHz}$, and clicks). Between 2 and 4 weeks significant changes were not found for any stimulus $(<5 \mathrm{~dB}$; Wilcoxon test, $p>0.05$ ).

The threshold change during the second week is shown as a function of the threshold shift at day 1 in Fig. 5. Two points arise from this figure. Firstly, both for $2 \mathrm{kHz}$



Fig. 3. CAP threshold shifts 7 days after deafening versus threshold shifts 1 day after deafening. (A) 2-kHz tones, (B) click stimuli. In three cases (all for clicks, B) the thresholds reached ceiling values: triangles indicate data above ceiling values after 7 days; the diamond indicates ceiling values for both 1 day and 7 days; the circles indicate the remaining regular data (thresholds below ceiling). Statistics for $2 \mathrm{kHz}: R^{2}=0.39, F(1,18)=11.5, p=0.003$; for clicks: $R^{2}=0.35, F(1,17)=9.3, p=0.007$.
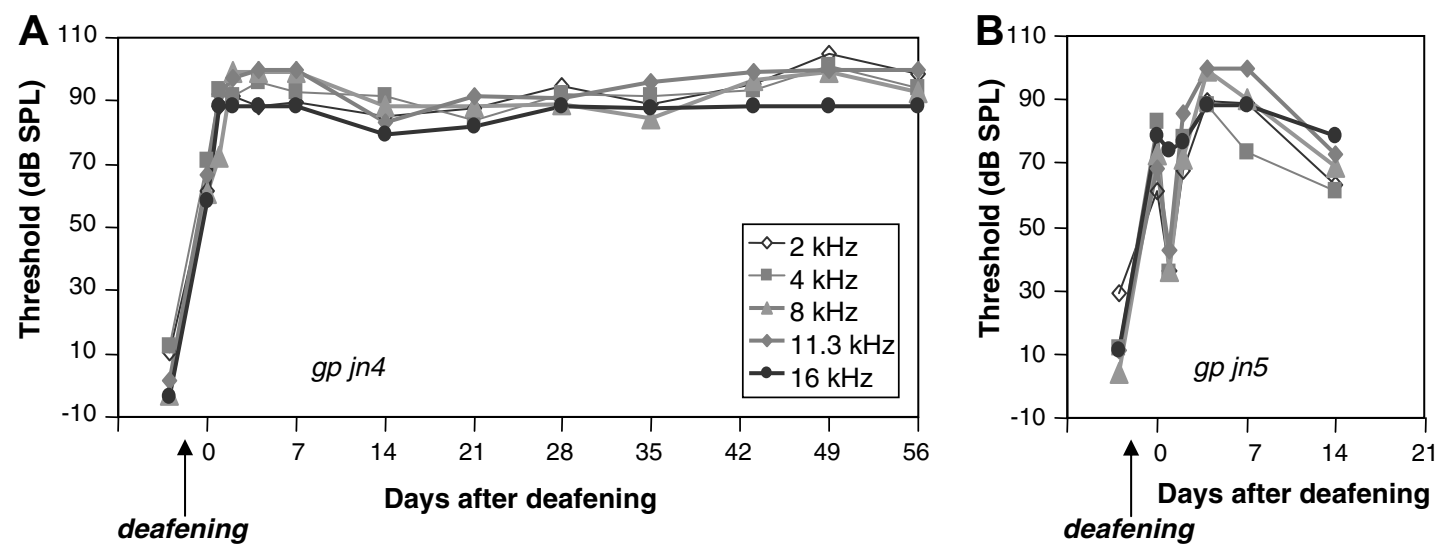

Fig. 4. Time courses of CAP thresholds up to 56 days (A) or 14 days (B) for five different tone frequencies (2-16 kHz) for two individual guinea pigs. The CAP thresholds before deafening were recorded on day 0 , just before the deafening surgery. In (A) thresholds were above ceiling values at several measurements, for instance for $8,11.3$ and $16 \mathrm{kHz}$ at day 4 , day 7 and day 49 . In (B) ceiling values were reached for 11.3 and $16 \mathrm{kHz}$ at days 4 and 7 . 
(Fig. 5A) and for clicks (Fig. 5B), the majority of threshold changes were negative, reflecting lower thresholds at day 14 than at day 7. Thirteen of fifteen animals that were monitored during 2 weeks (note that 5 of the initial 20 animals were prepared for histology at day 7) had high thresholds at day 7 (see Section 3.2). Wilcoxon statistics in these 13 animals shows that the thresholds significantly decreased for tones of 4 and $11.3 \mathrm{kHz}(p<0.05)$ and for clicks $(p<0.01)$. For clicks the threshold decreases were greater than $5 \mathrm{~dB}$ in 7 out of 12 animals. Secondly, close inspection of the data in Fig. 5 suggested that large second-week threshold decreases occurred in those animals which had
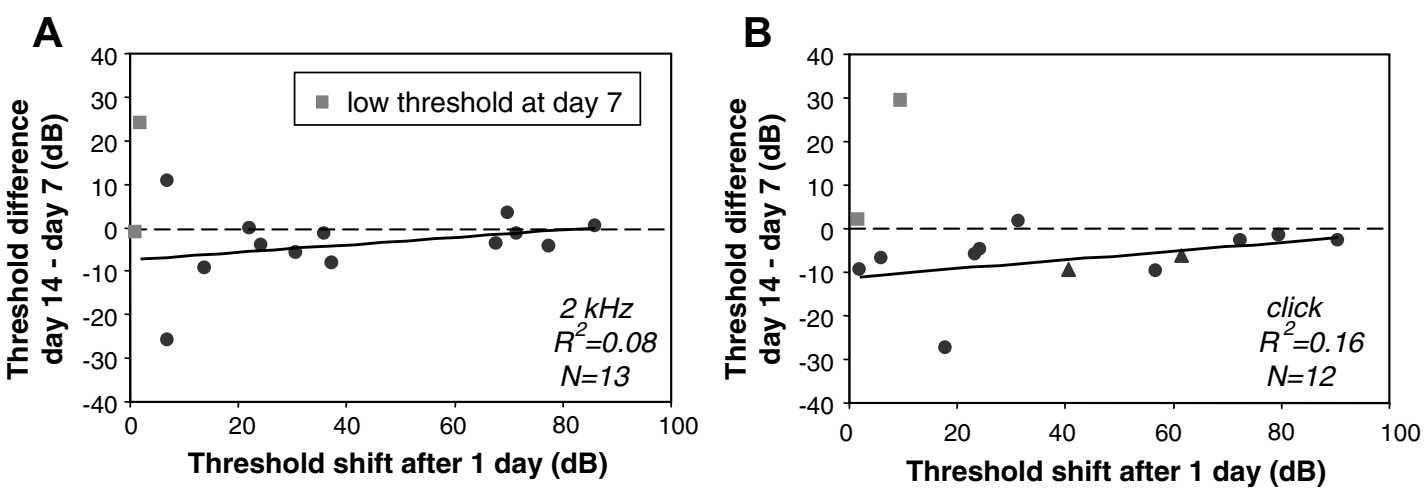

Fig. 5. The difference between thresholds at 14 and at 7 days after deafening versus threshold shifts 1 day after deafening. Negative values of the threshold difference reflect a lower threshold after 14 days than after 7 days (indicating recovery), positive values reflect the opposite (indicating decline). The grey squares indicate the guinea pigs with relatively low thresholds, the triangles indicate data with thresholds above the ceiling value on day 7 . Regression analysis is performed on all data apart from the low-threshold data (squares). (A) 2-kHz tones and (B) click stimuli.

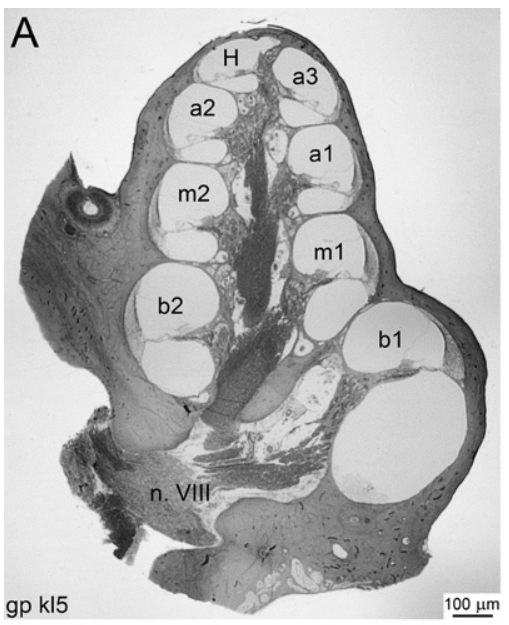

\begin{tabular}{ccc}
\hline Location & $\begin{array}{c}\text { Distance from } \\
\text { apex }(\mathrm{mm})\end{array}$ & CF $(\mathrm{kHz})$ \\
\hline b1 & 16.5 & 26.2 \\
b2 & 12.5 & 10.4 \\
m1 & 9.5 & 5.1 \\
m2 & 7.5 & 2.7 \\
a1 & 5.5 & 1.3 \\
a2 & 3.5 & 0.7 \\
a3 & 1.5 & 0.3 \\
\hline
\end{tabular}
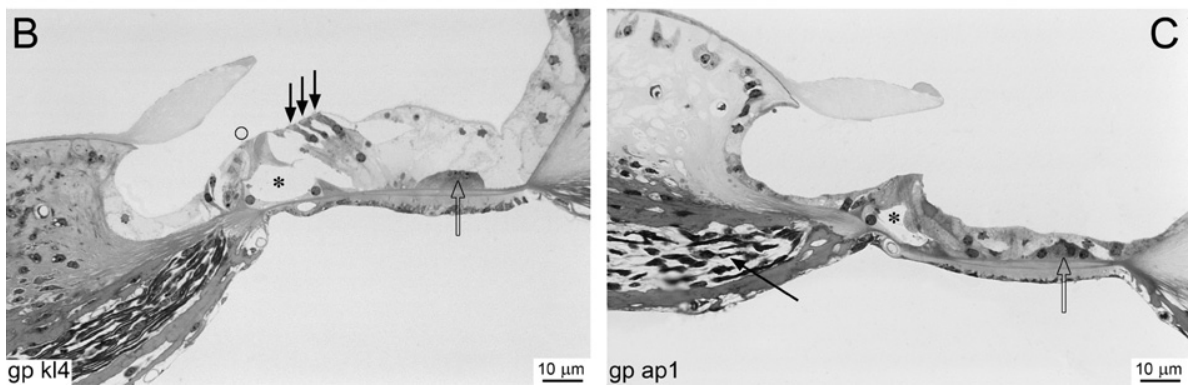

Fig. 6. (A) Midmodiolar section $(1 \mu \mathrm{m})$ of cochlea of guinea pig $k l 5$ ( 1 week after deafening). Transections of the cochlear half-turns are labeled b1 and b2 for the basal turns, $\mathrm{m} 1$ and $\mathrm{m} 2$ for the middle turns, and a1, a2, and a3 for the apical turns. The table (Van Ruijven et al., 2005) indicates the position along the basilar membrane and characteristic frequency $(\mathrm{CF})$ which correspond to the particular location of the transections. H: helicotrema; n.VIII: cochlear nerve. (B) Organ of Corti of normal cochlea of guinea pig kl4, in cochlear half-turn b1. o: inner hair cell; arrows: outer hair cells; asterisk: tunnel of Corti; open arrow: Boettcher's cells. (C) Organ of Corti in b1 8 weeks after deafening (guinea pig apl). The organ of Corti is collapsed with both the IHCs and OHCs missing. The amount of nerve fibers in the spiral osseous lamina (arrow) is clearly diminished. Asterisk: tunnel of Corti; open arrow: Boettcher's cells. 
relatively low thresholds one day after deafening. This correlation, however, was not significant (linear regression, $p>0.1)$.

The thresholds of the control animals remained stable over the course of 8 weeks with the final thresholds not significantly different from the initial thresholds (average changes $<4 \mathrm{~dB} ; p>0.2$ ).

\subsection{Hair cell survival}

Midmodiolar sections of the cochleas of deafened and control animals were examined with light microscopy (Fig. 6). Fig. 6B shows a normal organ of Corti and Fig. 6C shows a typical example of the effect of the combined treatment with kanamycin and furosemide on the morphology of the organ of Corti. The organ of Corti was collapsed with both the OHCs and IHCs missing. The amount of nerve fibers in the spiral osseous lamina was largely diminished.

The general effect of deafening on hair cell loss is reflected by the distribution of surviving hair cells along the basilar membrane (Fig. 7). On the average, OHC loss was complete in the basal turns and the percentage of surviving $\mathrm{OHC}$ increased along the basilar membrane towards the apical turns (Fig. 6A). In 12 animals no IHCs were found, in the eight remaining animals IHC survival was found in all cochlear turns. The hair cell survival did not significantly vary with the period after deafening treatment (Kruskal-Wallis test, $p>0.5$; IHC, $\mathrm{OHC}$ ).

The final CAP thresholds (recorded on the day of histological preparation of the cochlea) were significantly correlated to the surviving hair cells (linear regression, $p<0.01$ ) as shown in Fig. 7B and C. Multiple regression analysis showed that other factors such as duration after deafening and SGC packing density, were not significant $(p>0.5)$ in determining the CAP thresholds. Four animals showed a large HC survival (about 40-70\%). As expected, two out of these four were the animals that showed relatively low thresholds at day 7 (Fig. 3). One of the remaining two animals showed a relatively large recovery during the second week (animal jn5, see Fig. 4B).
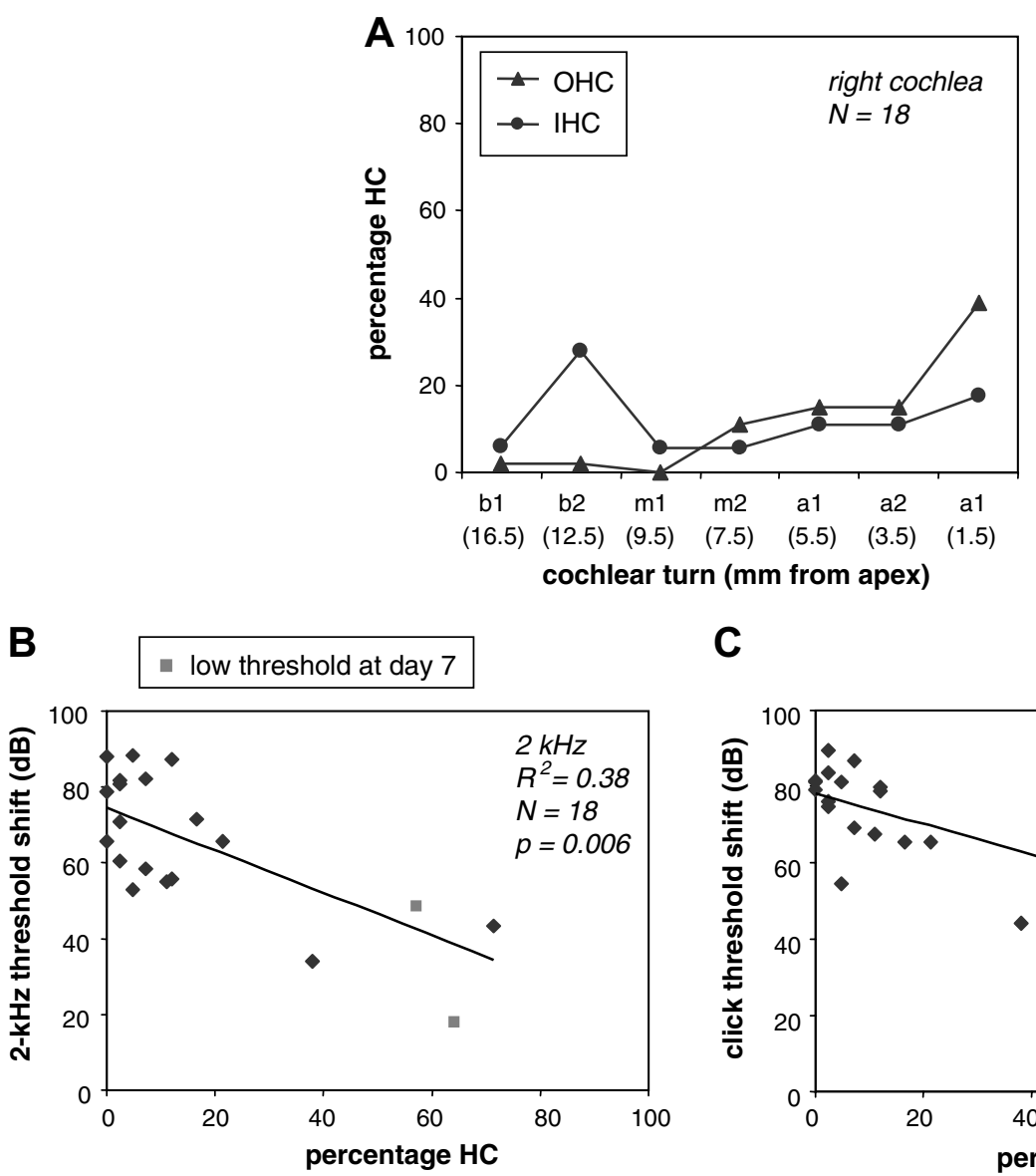

C

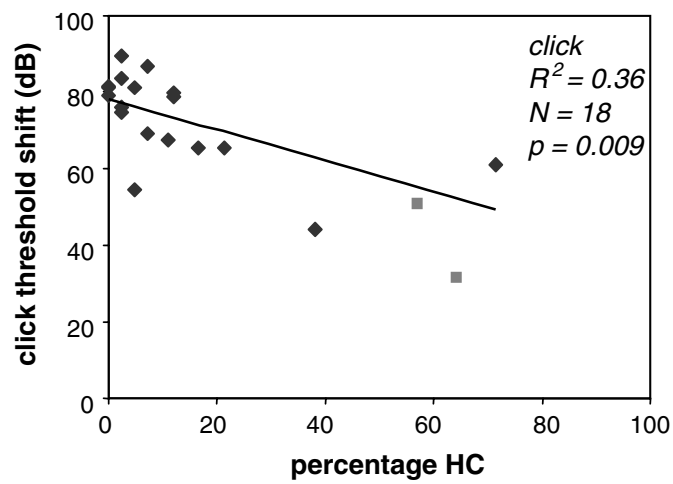

Fig. 7. (A) Hair cell survival averaged across animals with high thresholds at 7 days after deafening (threshold shift for clicks $>55 \mathrm{~dB} ; n=18$ ). OHC and IHC percentages are plotted separately as a function of cochlear half-turn. (B, C) CAP thresholds versus average HC survival. OHC and IHC numbers have been averaged across cochlear half-turns, and subsequently the obtained OHC and IHC percentages have been averaged into one data point. The grey squares indicate the guinea pigs with relatively low thresholds at 7 days after deafening (threshold shifts $<35 \mathrm{~dB}$ ). (B) Thresholds to 2 -kHz tones. For animals with high thresholds at 7 days $(n=18): R^{2}=0.38, F(1,16)=9.9, p=0.006$; for all animals $(n=20): R^{2}=0.56, F(1,18)=22.7, p<0.001$. $(\mathrm{C})$ Thresholds to click stimuli. For animals with high thresholds at 7 days: $R^{2}=0.36, F(1,16)=8.9, p=0.009$; for all animals: $R^{2}=0.59, F(1,18)=26.1$, $p<0.001$ 


\subsection{Spiral ganglion cell survival}

The light micrographs in Fig. 8 illustrate the progressive degeneration of SGCs with the period after the deafening treatment. Some loss of SGCs is visible 2 weeks after deafening; the loss is severe after 4 and 8 weeks.

The SGC packing density was measured in the three most basal half-turns (b1, b2, m1). Fig. 9 shows the time course of the SGC packing density measured in both cochleas of the normal-hearing animals $(n=5)$ and the deafened animals that showed high thresholds at day 7 $(n=18)$. The SGC packing density decreased with the period after deafening. This decrease can be described by an exponential decay function with a time constant of 7 weeks ( $1 / e=37 \%$ survival after 7 weeks).

Furthermore, the SGC packing density depended on the survival of IHCs. Fig. 10 shows the data separately for each survival time, indicating a modest increase of SGC density with IHC survival (increase of about 200 SGCs/ $\mathrm{mm}^{2}$ from $0 \%$ to $100 \%$ IHC). Multiple regression analysis with IHC percentage and duration after deafening as independent variables showed, apart from the strong correlation with the duration after deafening, that the SGC packing density was significantly correlated to the IHC survival $(p<0.05)$.

\subsection{CAP thresholds and hair cell survival}

Fig. 11A shows the thresholds at one day after deafening as a function of hair cell survival, comparable to Fig. 7B. The thresholds at one day after deafening appeared to show a relation to hair cell survival which could be best described by a power function $\left(R^{2}=0.60, p<0.0001\right)$. It might be attractive to predict hair cell survival on the basis of an early electrophysiological assessment. Therefore, hair cell survival is plotted as a function of the day-1 threshold shifts (Fig. 11B). The data show that for threshold shifts less than $3 \mathrm{~dB}$ hair cell survival is greater than $10 \%$, and for threshold shifts greater than $30 \mathrm{~dB}$ hair cell survival is less than $10 \%$.
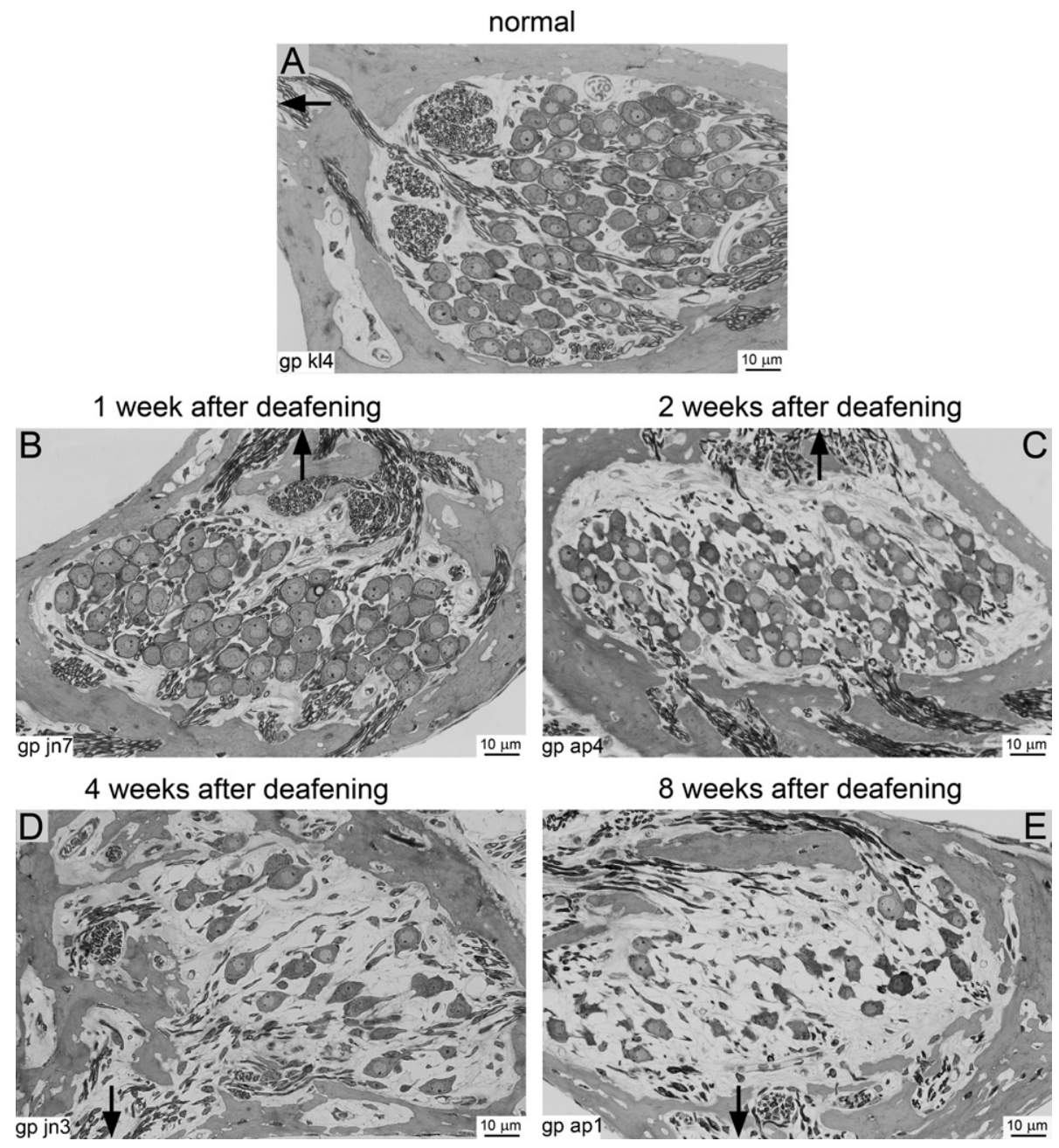

Fig. 8. Spiral ganglion cells in normal cochlea and in cochleas following concomitant kanamycin and furosemide administration. The arrows in the micrographs indicate the direction of the organ of Corti. (A) Normal cochlea, lower basal turn b1 (guinea pig $k l 4$ ). (B) One week after deafening (guinea pig $j n 7$ ). (C) Two weeks after deafening (guinea pig $a p 4$ ). (D) Four weeks after deafening (guinea pig jn3). (E) Eight weeks after deafening (guinea pig $a p 1$ ); (B)-(E): in lower middle turn $\mathrm{m} 1$. 


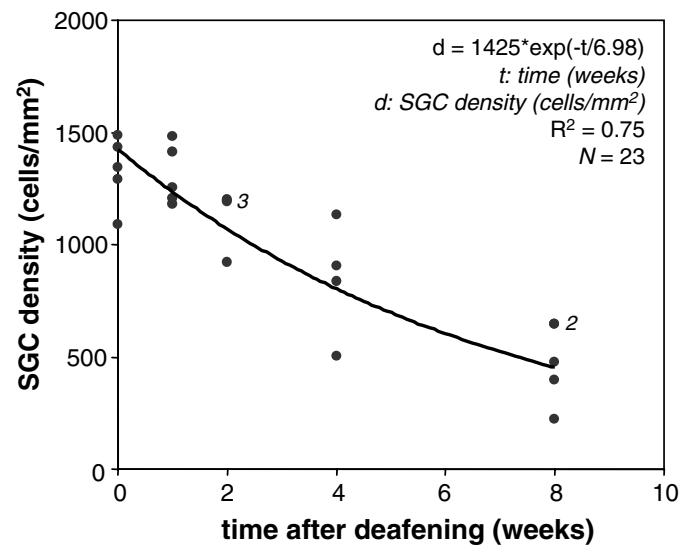

Fig. 9. SGC packing density as a function of survival time after deafening. The data include 5 normal-hearing animals and 18 deafened animals. Like the deafened animals, the normal control animals had an electrode implanted on the right cochlea. The SGC packing density is obtained by averaging SGC densities across the three most basal half-turns (b1, b2, $\mathrm{m} 1$ ), and by averaging over both ears. The italic digit near a data point indicates the number of overlapping data points. An exponential function has been fitted to the data: the time constant is 7.0 weeks, indicating that after that period $37 \%(1 / \mathrm{e})$ of the SGCs are still present. Linear regression of logarithm of SGC density versus survival time: $R^{2}=0.75$, $F(1,22)=64.6, p<0.0001$.

\section{Discussion}

The time courses of the CAP thresholds registered after the deafening procedure with standard doses of kanamycin and furosemide contained two interesting features. First, the threshold shifts at one day after deafening were highly variable among animals ( $100 \mathrm{~dB}$ range). Second, there was a small but significant recovery during the second week following substantial threshold shifts measured 4-7 days after deafening $(55-85 \mathrm{~dB})$.

\subsection{First-day thresholds}

The immediate threshold shifts we found after the deafening treatment agree with other studies applying administration of an aminoglycoside in combination with a loop diuretic (West et al., 1973; McFadden et al., 2004; Nourski et al., 2004). This early decline of the cochlear response is attributed to the reversible effect of furosemide, including the smaller effect on the $\mathrm{CM}$ than on the CAP (CAP: Sewell, 1984; Hu et al., 2003; CM: West et al., 1973; Van Emst et al., 1997). A drop of the endocochlear potential is thought to cause the decline of hair cell current $(\mathrm{CM})$ and cochlear nerve activity. Recording cochlear potentials, West et al. (1973), Nourski et al. (2004) and McFadden et al. (2004) found a recovery of the response within $1 \mathrm{~h}$, which can be attributed to a recovery of the endocochlear potential (Sewell, 1984; Van Emst et al., 1997). This recovery typically lasted only briefly (less than $4 \mathrm{~h}$ ) and, subsequently, a second decline of the response occurred. However, in some animals (two of six in Nourski et al.) the recovery lasted for at least $12 \mathrm{~h}$. The current findings indicate that the recovery may last for even $24 \mathrm{~h}$, in which case the response either strongly declines during the second 24-h period or it weakly declines and does not reach a high threshold (as was found in 2 out of 20 animals). Thus, the second phase of the threshold increase occurs within $5 \mathrm{~h}$, or between 24 and $48 \mathrm{~h}$. In the former case the threshold shift is always large ( $>30 \mathrm{~dB} /$ day), and in the latter case in the shift is large or small. A schematic of the three possible time courses in the first two days after deafening, combining the data of Nourski et al. and ours, is illustrated in Fig. 12. This second phase can be attributed to severe kanamycin-induced damage to OHCs as observed within $6 \mathrm{~h}$ by Russell et al. (1979). The high correlation we found between CM and CAP thresholds at day 1 also indicates that during the first day the main damage is inflicted upon the OHCs. In most animals, the thresholds reached a maximum after four days (Figs. 2A and B). This concurs with histological studies indicating that hair cell loss, which is initially limited to OHC loss, has stabilized in three to five days after deafening (Webster and Webster, 1981; Dodson, 1997).
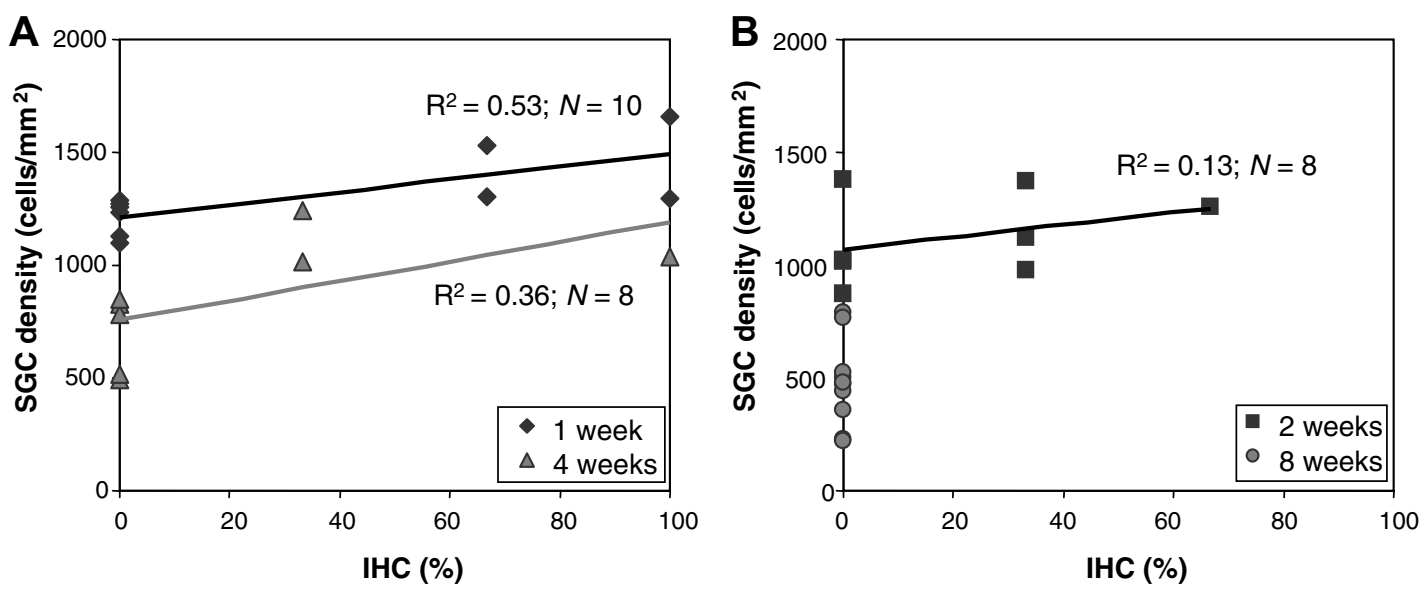

Fig. 10. SGC packing density as a function of percentage surviving IHCs. SGC packing density and IHC numbers have been averaged across the three most basal half-turns (b1, b2, m1). For clarity, the data are shown in two plots: 1 week and 4 weeks after deafening (A), 2 and 8 weeks after deafening (B). 

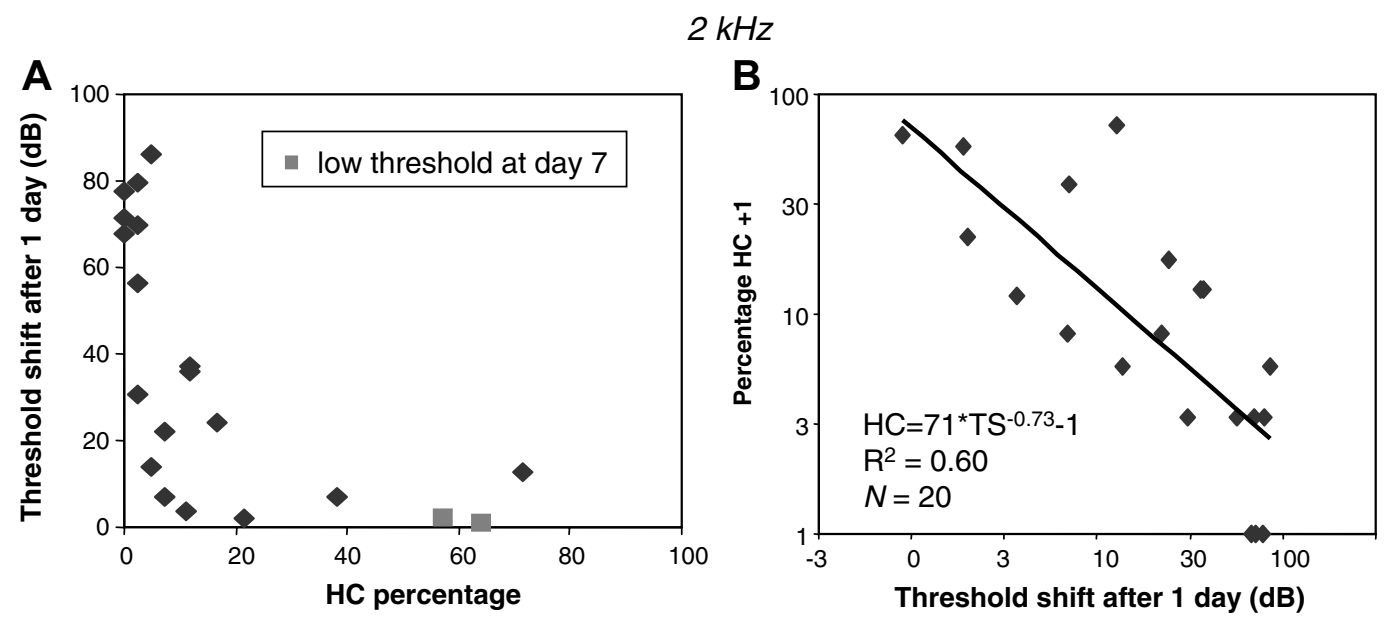

Fig. 11. Threshold shift after 1 day for $2-\mathrm{kHz}$ tones related to percentage surviving hair cells. OHC and IHC numbers have been averaged across all cochlear half-turns, and subsequently the obtained OHC and IHC percentages have been averaged. (A) Thresholds as a function of HC percentage, plotted as in Fig. 7B. (B) HC percentage as a function of thresholds, plotted on a double logarithmic scale. Linear regression: $R^{2}=0.60, F(1,18)=27.0$, $p<0.0001$.

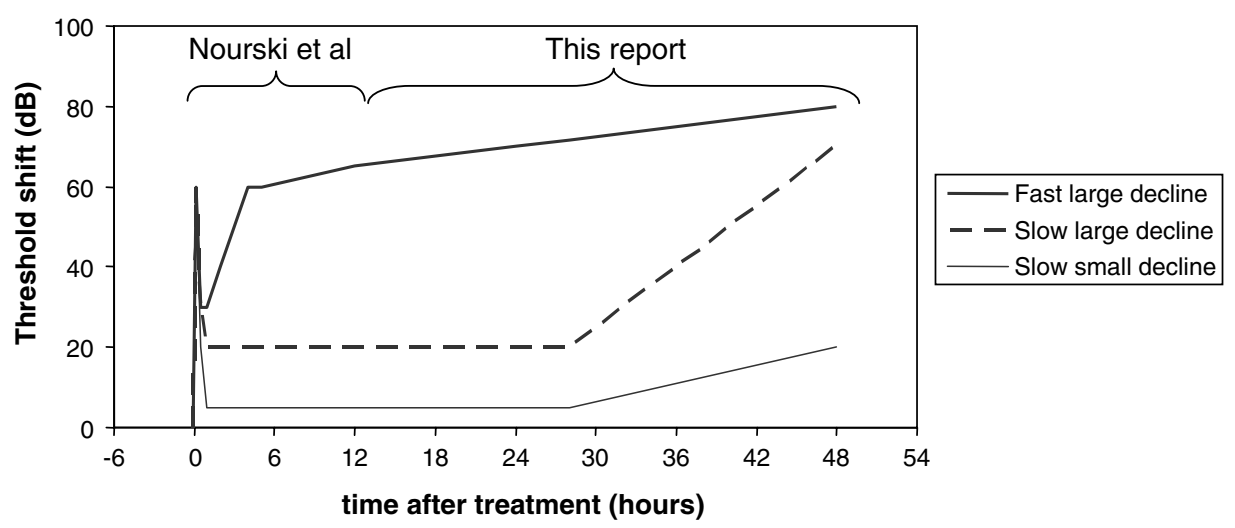

Fig. 12. Schematic of possible early time courses after the deafening procedure. The course during the first $12 \mathrm{~h}$ is based on data of Nourski et al. (2004) and the course between 24 and $48 \mathrm{~h}$ is based on the data presented in this report. The immediate threshold shift after deafening is found in both reports.

The wide distribution of threshold shifts at day 1 (Fig. 2D) can be explained by a variability in either the degree (in $\mathrm{dB}$ ) or the duration of the recovery. The former might contribute more than the latter, considering the lack of a correlation between threshold shifts and period after deafening (Fig. 2C).

The correlations between CAP thresholds after 7 days and those after 1 day (Fig. 3) and between surviving hair cells and CAP thresholds after 1 day (Fig. 11) imply that the faster the kanamycin reaches its cellular target the larger the final effect. The relatively high correlation between hair cell survival and thresholds after 1 day, in spite of the long period between histology and recording, might be surprising. However, one should consider the distribution of threshold shifts at day 1 which is uniform and wide compared to the later distributions (Figs. 2 and 3). The measuring errors can be assumed to be constant over time, however, the measuring error relative to the spread of thresholds is smaller at day 1 than at later moments. This small relative error might counterbalance the effect of the early timing of the measurement. In other words, animals can be distinguished on the basis of the thresholds at day 1 no worse than on the basis of thresholds measured later. It allows for a fair assessment after only one day of the long-term effect of the deafening treatment.

\subsection{Second-week recovery}

Various studies using the deafening method according to West et al. (1973) apply an inclusion criterion based on absolute thresholds or threshold shifts $(50-60 \mathrm{~dB})$ after 4-7 days (Mitchell et al., 1997; Kanzaki et al., 2002; Wise et al., 2005; Shepherd et al., 2005). According to those criteria 18 out of 20 guinea pigs in our study had sufficient threshold shifts ( $>55 \mathrm{~dB}$ for clicks). With such high thresholds, which indicate severe damage to the organ of Corti, a recovery might not be likely. However, our data strongly suggest some recovery between 7 and 14 days after deafening (Figs. 4 and 5). Similarly, in a longitudinal CAP study in guinea pigs Aran and Darrouzet (1975) found recovery 
from kanamycin treatment after 8-10 days, also in the second week. They attributed the recovery to clearance of kanamycin from the cochlea. Alternatively, the ototoxic drug might hamper strial function, thereby reducing the endocochlear potential (EP). Recovery of the EP would improve the function of the surviving hair cells. Klis et al. (2002) presented evidence for this mechanism to explain recovery from a $60 \mathrm{~dB}$ hearing loss after cisplatin treatment.

This late functional recovery implies that caution should be taken in studies in which total hair cell loss is required. In that case, the threshold shift after 7 days or earlier is not an appropriate inclusion criterion and thresholds after at least 2 weeks or the post hoc histological examination of the organ of Corti might be used instead. Treatment during the second week with for instance neural growth factors aimed at enhancing SGC survival, might be confounded by the spontaneous functional recovery. In particular, in studies with treatment to induce hair cell regeneration one should be aware of, and preferably avoid, the possibility of spontaneous recovery not related with the treatment.

The slow decline following the second-week recovery (Fig. 4A) is reminiscent of data reported by Aran and Darrouzet (1975). They found a deterioration phase two months after the start of the recovery phase (discussed above). Similarly, Stengs et al. (1997) reported some deterioration of cochlear potentials 2-4 months after recovery from cisplatin-induced hearing loss in guinea pigs.

\section{3. $S G C$ degeneration}

The degeneration of SGC after cochlear trauma induced by aminoglycosides has been studied extensively (Ylikoski et al., 1974; Webster and Webster, 1981; Xu et al., 1993; Dodson, 1997; Leake and Rebscher, 2004). It is generally thought that the degeneration of SGCs is secondary to loss of IHCs and the afferent nerve terminal, since SGC trauma has never been observed in the presence of IHCs. Conditions in which OHCs are lost while IHCs are intact do not lead to SGC loss (Ylikoski et al., 1974). The correlations between SGC packing density and IHC percentage (Fig. 10) are congruent with this proposition. Various mechanisms might play a role in the degeneration such as absence of neurotrophic support, absence of neural activity, and glutamate excitotoxicity (discussed in Dodson, 1997).

In global terms, the time course we found (Fig. 9) concurs with findings of others. The time constant varies between studies depending on various factors such as species and type and/or dosage of insult (e.g., Dodson and Mohuiddin, 2000; Leake and Rebscher, 2004; McFadden et al., 2004). For instance, a time constant of about 3 weeks has been found in guinea pigs deafened by an intracochlear injection with gentamicin (Dodson and Mohuiddin, 2000) and a time constant of about 8 months has been found in cats neonatally deafened with neomycin (Leake and Rebscher, 2004); in humans the degeneration is generally even slower. The shape of the time course might vary as well. We chose to fit the data with an exponential function with a single time constant. Data obtained over longer periods than in the current study ( 8 weeks) indicate that a single time constant does not suffice (Leake and Rebscher, 2004, in cat, study of 7 years; Dodson and Mohuiddin, 2000 , in guinea pig, study of 7 months). As various mechanisms might play a role in the degeneration (see above), multiple time constants are plausible.

\subsection{Implications}

The recordings at day 1 are important to predict final thresholds and hair cell survival. If very limited or no survival is required, e.g. for regeneration research, then the first-day threshold should be high $(>30 \mathrm{~dB}$ for $2 \mathrm{kHz}$ tones). On the other hand, if some residual hearing is needed, e.g., in the context of the combination of electrical and acoustical stimulation, then thresholds should be low. In general, the thresholds at day 1 can serve as a useful independent variable.

\section{Acknowledgements}

This study was supported by the Heinsius-Houbolt Foundation. We are grateful to Rik Mansvelt Beck for producing the electrodes, Ferry Hendriksen for assisting with histology, Kelly Maijoor for assisting with surgery and recordings, and Frank Hamers for the recording and analysis software.

\section{References}

Aran, J.M., Darrouzet, J., 1975. Observation of click-evoked compound VIII nerve responses before, during, and over seven months after kanamycin treatment in the guinea pig. Acta Otolaryngol. 79, 24-32.

De Groot, J.C.M.J., Veldman, J.E., Huizing, E.H., 1987. An improved fixation method for guinea pig cochlear tissues. Acta Otolaryngol. 104, 234-242.

Dodson, H.C., 1997. Loss and survival of spiral ganglion neurons in the guinea pig after intracochlear perfusion with aminoglycosides. J. Neurocytol. 26, 541-556.

Dodson, H.C., Mohuiddin, A., 2000. Response of spiral ganglion neurones to cochlear hair cell destruction in the guinea pig. J. Neurocytol. 29, 525-537.

Gillespie, L.N., Clark, G.M., Bartlett, P.F., Marzella, P.L., 2003. BDNFinduced survival of auditory neurons in vivo: cessation of treatment leads to accelerated loss of survival effects. J. Neurosci. Res. 71, 785790.

Hamers, F.P.T., Wijbenga, J., Wolters, F.L.C., Klis, S.F.L., Sluyter, S., Smoorenburg, G.F., 2003. Cisplatin ototoxicity involves organ of Corti, stria vascularis and spiral ganglion: modulation by alphaMSH and ORG 2766. Audiol. Neurootol. 8, 305-315.

Hu, N., Abbas, P.J., Miller, C.A., Robinson, B.K., Nourski, K.V., Jeng, F.-C., Abkes, B.A., Nichols, J.M., 2003. Auditory response to intracochlear electric stimuli following furosemide treatment. Hear. Res. 185, 77-89.

Izumikawa, M., Minoda, R., Kawamoto, K., Abrashkin, K.A., Swiderski, D.L., Dolan, D.F., Brough, D.E., Raphael, Y., 2005. Auditory hair cell replacement and hearing improvement by Atoh1 gene therapy in deaf mammals. Nat. Med. 11, 271-276. 
Kanzaki, S., Stover, T., Kawamoto, K., Prieskorn, D.M., Altschuler, R.A., Miller, J.M., Raphael, Y., 2002. Glial cell line-derived neurotrophic factor and chronic electrical stimulation prevent VIII cranial nerve degeneration following denervation. J. Comp. Neurol. 454, 350360 .

Klis, S.F.L., O'Leary, S.J., Hamers, F.P.T., de Groot, J.C.M.J., Smoorenburg, G.F., 2000. Reversible cisplatin ototoxicity in the albino guinea pig. NeuroReport 11, 623-626.

Klis, S.F.L., O’Leary, S.J., Wijbenga, J., de Groot, J.C.M.J., Hamers, F.P.T., Smoorenburg, G.F., 2002. Partial recovery of cisplatin-induced hearing loss in the albino guinea pig in relation to cisplatin dose. Hear. Res. 164, 138-146.

Leake, P.A., Rebscher, S.J., 2004. Anatomical considerations of electrical stimulation. In: Zeng, F.-G., Popper, A.N., Fay, R.R. (Eds.), Cochlear Implants: Auditory Prostheses and Electric Hearing. Springer, New York, pp. 101-148.

Lousteau, R.J., 1987. Increased spiral ganglion cell survival in electrically stimulated, deafened guinea pig cochleae. Laryngoscope 97, 836-842.

McFadden, S.L., Ding, D., Jiang, H., Salvi, R.J., 2004. Time course of efferent fiber and spiral ganglion cell degeneration following complete hair cell loss in the chinchilla. Brain Res. 997, 40-51.

Miller, J.M., Chi, D.H., O'Keeffe, L.J., Kruszka, P., Raphael, Y., Altschuler, R.A., 1997. Neurotrophins can enhance spiral ganglion cell survival after inner hair cell loss. Int. J. Dev. Neurosci. 15, 631-643.

Mitchell, A., Miller, J.M., Finger, P.A., Heller, J.W., Raphael, Y., Altschuler, R.A., 1997. Effects of chronic high-rate electrical stimulation on the cochlea and eighth nerve in the deafened guinea pig. Hear. Res. 105, 30-43.

Nourski, K.V., Miller, C.A., Hu, N., Abbas, P.J., 2004. Co-administration of kanamycin and ethacrynic acid as a deafening method for acute animal experiments. Hear. Res. 187, 131-133.

Russell, N.J., Fox, K.E., Brummett, R.E., 1979. Ototoxic effects of the interaction between kanamycin and ethacrynic acid. Cochlear ultrastructure correlated with cochlear potentials and kanamycin levels. Acta Otolaryngol. 88, 369-381.

Sewell, W.F., 1984. The effects of furosemide on the endocochlear potential and auditory-nerve fiber tuning curves in cats. Hear. Res. 14, 305-314.
Shepherd, R.K., Coco, A., Epp, S.B., Crook, J.M., 2005. Chronic depolarization enhances the trophic effects of brain-derived neurotrophic factor in rescuing auditory neurons following a sensorineural hearing loss. J. Comp. Neurol. 486, 145-158.

Staecker, H., Kopke, R., Malgrange, B., Lefebvre, P., Van De Water, T.R., 1996. NT-3 and/or BDNF therapy prevents loss of auditory neurons following loss of hair cells. Neuroreport 7, 889-894.

Stengs, C.H.M., Klis, S.F.L., Huizing, E.H., Smoorenburg, G.F., 1997. Cisplatin-induced ototoxicity; electrophysiological evidence of spontaneous recovery in the albino guinea pig. Hear. Res. 111, 103-113.

Van Emst, M.G., Klis, S.F., Smoorenburg, G.F., 1997. Identification of the nonlinearity governing even-order distortion products in cochlear potentials. Hear. Res. 114, 93-101.

Van Ruijven, M.W.M., de Groot, J.C.M.J., Smoorenburg, G.F., 2004. Time sequence of degeneration pattern in the guinea pig cochlea during cisplatin administration. A quantitative histological study. Hear. Res. 197, 44-54.

Van Ruijven, M.W.M., de Groot, J.C.M.J., Klis, S.F.L., Smoorenburg, G.F., 2005. The cochlear targets of cisplatin: An electrophysiological and morphological time-sequence study. Hear. Res. 205, 241-248.

Webster, M., Webster, D.B., 1981. Spiral ganglion neuron loss following organ of Corti loss: a quantitative study. Brain Res. 212, 17-30.

West, B.A., Brummett, R.E., Himes, D.L., 1973. Interaction of kanamycin and ethacrynic acid. Severe cochlear damage in guinea pigs. Arch. Otolaryngol. 98, 32-37.

Wise, A.K., Richardson, R., Hardman, J., Clark, G., O'Leary, S., 2005. Resprouting and survival of guinea pig cochlear neurons in response to the administration of the neurotrophins brain-derived neurotrophic factor and neurotrophin-3. J. Comp. Neurol. 487, $147-165$.

Xu, S.-A., Shepherd, R.K., Chen, Y., Clark, G.M., 1993. Profound hearing loss in the cat following the single co-administration of kanamycin and ethacrynic acid. Hear. Res. 70, 205-215.

Ylikoski, J., Wersäll, J., Björkroth, B., 1974. Degeneration of neural elements in the cochlea of the guinea-pig after damage to the organ of Corti by ototoxic antibiotics. Acta Otolaryngol. Suppl. $326,23-41$. 